\title{
The Role of Citrate Transporter INDY in Metabolism and Stem Cell Homeostasis
}

\author{
Kavitha Kannan ${ }^{1}$ and Blanka Rogina ${ }^{1,2, *(\mathbb{D}}$ \\ 1 Department of Genetics \& Genome Sciences, School of Medicine, University of Connecticut Health Center, \\ Farmington, CT 06030, USA; kkannan@uchc.edu \\ 2 Institute for Systems Genomics, School of Medicine, University of Connecticut Health Center, \\ Farmington, CT 06030, USA \\ * Correspondence: Rogina@uchc.edu; Tel.: +1-860-679-8771
}

Citation: Kannan, K.; Rogina, B. The Role of Citrate Transporter INDY in Metabolism and Stem Cell

Homeostasis. Metabolites 2021, 11, 705 https://doi.org/10.3390/

metabo11100705

Academic Editor: James McCullagh

Received: 13 September 2021

Accepted: 12 October 2021

Published: 15 October 202

Publisher's Note: MDPI stays neutral with regard to jurisdictional claims in published maps and institutional affiliations.

Copyright: (C) 2021 by the authors Licensee MDPI, Basel, Switzerland This article is an open access article distributed under the terms and conditions of the Creative Commons Attribution (CC BY) license (https:// creativecommons.org/licenses/by/ $4.0 /)$.

\begin{abstract}
I'm Not Dead Yet (Indy) is a fly gene that encodes a homologue of mammalian SLC13A5 plasma membrane citrate transporter. Reducing expression of Indy gene in flies, and its homologues in worms, extends longevity. Indy reduction in flies, worms, mice and rats affects metabolism by regulating the levels of cytoplasmic citrate, inducing a state similar to calorie restriction. Changes include lower lipid levels, increased insulin sensitivity, increased mitochondrial biogenesis, and prevention of weight gain, among others. The INDY protein is predominantly expressed in fly metabolic tissues: the midgut, fat body and oenocytes. Changes in fly midgut metabolism associated with reduced Indy gene activity lead to preserved mitochondrial function and reduced production of reactive oxygen species. All these changes lead to preserved intestinal stem cell homeostasis, which has a key role in maintaining intestinal epithelium function and enhancing fly healthspan and lifespan. Indy gene expression levels change in response to caloric content of the diet, inflammation and aging, suggesting that INDY regulates metabolic adaptation to nutrition or energetic requirements by controlling citrate levels.
\end{abstract}

Keywords: Indy; SLC13A5; aging; intestinal stem cells; citrate transporter; longevity gene; metabolism

\section{Introduction}

I'm not dead yet (Indy) is a fly homologue of mammalian SLC13A5 plasma membrane transporter with highest affinity for transporting the metabolite citrate, which is a critical regulator of metabolism [1-3]. Indy homologues have been identified in bacteria, worms, mice, rats, dogs, rabbits, monkeys, chimpanzees, zebrafish, pigs and humans [4-6]. The mammalian protein mINDY (also referred to as $\mathrm{NaCT}$ or $\mathrm{Na}^{+}$coupled citrate transporter) is a plasma membrane citrate transporter similar to Drosophila INDY, but requires $\mathrm{Na}^{+}$ as a co-transporter $[2,3,7,8]$. The structural and functional characteristics of mouse and human mINDY are also distinct: the active sites that bind citrate and $\mathrm{Na}^{+}$are different, and functionally, mouse mINDY is a low-capacity/high-affinity transporter, while human mINDY is a high-capacity/low-affinity transporter [9]. While differences exist between species, citrate transport is a conserved role for INDY. Citrate is a key metabolite in the tricarboxylic acid (TCA) cycle and has a highly conserved role in glucose, lipid, and energy metabolism across species. This is illustrated by studies that show similar effects of Indy reduction on metabolism in flies, worms, mice, rats, non-human primates and humans [10-12]. Reducing Indy gene expression levels extends longevity in flies and worms by affecting multiple metabolic pathways [1,10,13-17]. In this review, we focus on the effects of reduced INDY activity on metabolism, mitochondrial biogenesis and fly stem cell homeostasis that promote health and longevity. Table 1 includes abbreviations used in this review. 
Table 1. List of abbreviations.

\begin{tabular}{|c|c|}
\hline Abbreviation & Description \\
\hline ACLY & ATP citrate lyase \\
\hline AhR & Aryl hydrocarbon receptor \\
\hline AMPK & $5^{\prime} \mathrm{AMP}$-activated protein kinase \\
\hline CeNac2 & Caenorhabditis elegans sodium cotransporter 2 \\
\hline CIC & Citrate/isocitrate carrier also known as SLC25A1 \\
\hline CR & Calorie restriction \\
\hline CREB & cAMP responsive element-binding protein \\
\hline DILPs & Drosophila Insulin-like peptides \\
\hline EB & Enteroblast \\
\hline EC & Enterocyte \\
\hline $\mathrm{EE}$ & Entero-endocrine cell \\
\hline ETC & Electron transport chain \\
\hline FoxO & Transcription factor from the Forkhead box-O family \\
\hline GstD5 & Glutathione S transferase D5 \\
\hline GstE1 & Glutathione S transferase E1 \\
\hline HepG2 & Hepatoma G2 cell line \\
\hline Hoppel & Transposable insertion variant \\
\hline IIS & Insulin/insulin-like growth factor signaling pathway \\
\hline IL-6 & Interleukin 6 \\
\hline Indy & Drosophila I'm not dead yet gene \\
\hline ISC & Intestinal stem cell \\
\hline mINDY & Mammalian homolog of Indy (also referred as mSLC13A5/NaCT) \\
\hline$m S L C 13 A 5$ & $\begin{array}{c}\text { Mammalian Sodium-dicarboxylate cotransporter solute carrier family } \\
13 \text {, member } 5 \text { (also referred as } m I N D Y / N a C T \text { ) }\end{array}$ \\
\hline $\mathrm{NaCT}$ & $\begin{array}{l}\text { Sodium }\left(\mathrm{Na}^{+}\right) \text {coupled citrate transporter (also referred as } \\
\text { mSLC13A5; mINDY) }\end{array}$ \\
\hline NAFLD & Non-alcoholic fatty liver disease \\
\hline dPGC-1/spargel & $\begin{array}{l}\text { Drosophila homologue of peroxisome proliferator-activated receptor } \\
\text { gamma co-activator-1 (PGC-1) }\end{array}$ \\
\hline PXR & Pregnane $X$ receptor \\
\hline ROS & Reactive oxygen species \\
\hline SLC13A5 & $\begin{array}{l}\text { Sodium-dicarboxylate cotransporter solute carrier family } 13 \text {, member } \\
5 \text { (also referred as INDY/NaCT) }\end{array}$ \\
\hline STAT3 & Signal transducer and activator of transcription 3 \\
\hline TCA & Tricarboxylic acid cycle \\
\hline VM & Visceral muscle \\
\hline
\end{tabular}

\section{Indy Reduction Extends Longevity of Flies and Worms by Mimicking Calorie Restriction}

In flies, the INDY protein is predominantly expressed on the plasma membrane of metabolic tissues - the midgut, fat body, and oenocytes - all of which play an important role in uptake and storage of nutrients and energy production [1,2]. The worm homologues of INDY are predominantly expressed in the intestinal tract [15]. Mammalian mINDY is expressed at high levels in liver, brain, and testis, while low levels are found in heart, 
kidney, bones, and ovary in humans, mice, and rats [10,18-20]. The primary source of citrate comes from citrate synthesis in the mitochondria. However, in tissues where INDY is expressed, extracellular citrate is transported into cells through INDY, and regulation of this transport is important for maintenance of cytoplasmic citrate homeostasis [19,21].

Reduced expression of Indy in fruit flies or its homologue CeNAC-2 in worms extends longevity $[1,13,14,16,17,22]$. Longevity in flies was extended in a number of independent Indy alleles, in which Indy mRNA levels are reduced by insertion of a P-element or a GFP protein trap in the first intron of the Indy gene $[1,13,16,22,23]$. Precise excision of the P-element in several Indy alleles resulted in a reversion to lifespan of control flies, confirming a causal relationship between P-element insertion in the Indy gene and fly longevity [1] The presence of the P-element in the first intron of the Indy gene decreases the levels of Indy mRNA and protein levels, most likely by affecting transcription. In addition, natural populations of flies heterozygous for a Hoppel transposon insertion variant in the first intron of the Indy gene have reduced INDY levels and extended lifespan [24]. Three of these lines examined in detail were from Oahu, Hawaii (collected in 1955), Captain Cook, Hawaii (collected in 2007), and Hidalgo, Mexico (collected in 2005). Thus, heterozygosity for the Hoppel element has been preserved in laboratory conditions for many years conferring advantage of reduced Indy levels including increased fecundity [24]. Original studies using five independent Indy alleles, including Ind $y^{206} /+$, Indy $y^{302} /+$, and Indy $y^{159} /+$, showed longevity extension between 80 and $100 \%$ in both mean and maximal lifespan. Additional studies using Indy $206 /+$ showed that Indy reduction extends lifespan in different genetic backgrounds of flies including wild-type Canton-S stock, the Hyperkinetic, Shaker, drop-dead stocks, and short- and long-lived lines of Luckinbill $[1,13,25]$. Each of these stocks were separately maintained from the wild-type stocks for $45-50$ years. Interestingly, Indy reduction further increases longevity of long-lived Luckinbill lines $[13,26]$. One of the original Indy ${ }^{206}$ alleles was treated with tetracycline to avoid any influence of Wolbachia on lifespan and backcrossed into control $y w$ and $w^{1118}$ genetic stocks for 10 generations. Compared to control stocks, longevity of Indy ${ }^{206}$ allele was extended in yw background, but not in $w^{1118}$ background $[13,22,27]$. The influence of genetic background on longevity is well-known, as the effect of Indy reduction on longevity in Drosophila has been examined and documented in varied genetic backgrounds listed in the above mentioned studies [1,13,16,22]. Genetic background has been shown to impact quantitative physiological traits measured as markers of aging as well as intestinal stem cell proliferation in Drosophila [28,29]. Use of a diet similar to calorie restriction (CR) may affect longevity of Indy alleles in the Toivonen et al. study [22,27]. Studies using different Indy alleles (Indy ${ }^{159}$, Indy ${ }^{E P 3044}$, Indy ${ }^{E P 3366}$, Indy ${ }^{E Y 01442}$, Indy EY01458, Indy EY013297, Indy KG07717) backcrossed into the $y w$ background showed that longevity extension is linked to the levels of Indy mRNA. A maximal increase in longevity of $34 \%$ was observed in heterozygous Indy $y^{206} /+$, Indy $159 /+$ and Indy ${ }^{Y C} 0030 /+$ alleles, in which Indy mRNA levels are about 55\% lower compared to controls [13]. A small reduction of Indy mRNA leads to about $20 \%$ longevity extension, while a non-significant reduction of Indy mRNA levels does not significantly affect Indy ${ }^{E P 3366 /+~ l i f e s p a n ~ c o m p a r e d ~ t o ~ c o n t r o l s . ~}$

Indy reduction regulates cytoplasmic citrate levels, which affects cellular metabolism and energy production in mitochondria. Reducing Indy expression lowers available energy resources and creates a state similar to CR. Accordingly, flies with reduced Indy levels have several metabolic and physiological phenotypes parallel to CR [30,31]. These changes include increased mitochondrial biogenesis, increased spontaneous physical activity, increased resistance to stress, reduced reactive oxygen species (ROS) production, smaller body weight and reduced insulin/insulin-like growth factor signaling (IIS), among others $[16,22,23,32]$. IIS is an evolutionarily conserved pathway involved in growth and metabolism. Reduced IIS extends lifespan in multiple species [33-35]. In Drosophila, the IIS pathway is activated under physiological conditions when nutrients are abundant and Drosophila insulin-like peptides (DILPs 1-8) bind to the insulin receptor (InR), which activates the downstream members of the IIS pathway. Activation of IIS results in phosphorylation of a transcription factor from the Forkhead box-O family (FoxO), which prevents 
its nuclear localization [36,37]. Low nutrient levels downregulate IIS, which leads to nuclear localization of unphosphorylated FoxO where it activates transcription of antioxidant genes and APGC-1/spargel, a Drosophila homologue of peroxisome proliferator-activated receptor gamma co-activator-1 (PGC-1). IIS is reduced under CR conditions and in Indy ${ }^{206} /+$ flies [16,22]. This is illustrated by findings that control flies on a CR diet and Indy ${ }^{206} /+$ flies on a high calorie diet have FoxO predominantly localized in the nuclei of fly fat bodies, which is indicative of reduced IIS [22,38]. In addition, the levels of dilp2, dilp3, and dilp5 were reduced in the heads and thoraces of $I n d y^{206} /+$ flies to identical levels found in CR flies [22]. Similarly, increased insulin sensitivity was found in $m I N D Y^{-} /{ }^{-}$knockout ( $m I N D Y-K O)$ mice, suggesting conserved INDY effects on insulin signaling and metabolic regulation [18].

Moreover, the longevity of Indy $206 /+$ heterozygous flies is not further extended by CR, which is consistent with Indy $y^{206} /+$ flies already being in a state of CR when kept on a regular diet [22,23]. While Indy ${ }^{206} /$ Indy ${ }^{206}$ homozygous flies live longer than controls on a regular and a high calorie diet, they live shorter on a CR diet, most likely due to starvation. Consistent with physiological changes in CR, studies in worms with reduced Indy homologue, CeNAC-2 show reduced body fat content and body size [14,17]. Likewise, mINDY-KO mice have decreased body weight, increased hepatic mitochondrial biogenesis, increased mitochondrial density, increased insulin sensitivity, higher lipid oxidation, and similar hepatic transcriptional changes to those found in liver of CR mice $[10,18]$. In rats, a reduction of mIndy levels with antisense oligonucleotides protects animals from harmful effects of diet-induced hepatic steatosis and insulin resistance [39,40]. Identification of similar phenotypes and changes associated with reduced INDY levels in multiple organisms suggests a highly conserved role of INDY in metabolism.

\section{The Role of INDY in Metabolism}

In flies, INDY is a plasma membrane transporter for TCA cycle intermediates [2]. Functional studies in frog oocytes show that INDY has a role as an anion exchanger, and exchanges citrate-citrate, citrate-succinate, citrate-oxaloacetate, but also succinate- $\alpha$ ketoglutarate, and succinate-fumarate [2]. Taken together, fly INDY is an exchanger of dicarboxylate and tricarboxylate TCA-cycle intermediates. Exchange of internal anion for external citrate is $\mathrm{pH}$-dependent but Na-independent. Thus, INDY transports citrate bidirectionally between the midgut and hemolymph (hemolymph is analogous to blood that acts as a circulatory fluid in flies). Citrate in hemolymph circulates to other metabolic tissues, mainly the fat body and oenocytes. In mammals, mINDY transports citrate from circulation across the plasma membrane, dominantly in the liver, brain, and testis [11,41]. In liver, mINDY is present in the sinusoidal membrane facing the blood [11,41]. INDY levels regulate glucose and lipid metabolism and energy production by affecting cytoplasmic citrate levels (Figure 1).

Cytosolic citrate is broken down to acetyl-CoA and oxaloacetate by the enzyme ATP citrate lyase (ACLY). Cytoplasmic acetyl-CoA can be used as a building block for fatty acids, triacylglycerols, cholesterol, and low-density lipoprotein synthesis [42-44]. When citrate is metabolized to malate, it enters mitochondria where it is used in the TCA cycle for generation of NADH and $\mathrm{FADH}_{2}$, which can enter the mitochondrial matrix for energy production. When ATP production is high, mitochondrial citrate is transported by mitochondrial citrate transporter CIC (Citrate/isocitrate carrier; also known as SLC25A1) into the cytosol in exchange for malate from cytoplasm into the matrix $[45,46]$. Under physiological conditions when required ATP levels are met, citrate can be used in catabolism. Cytoplasmic citrate levels affect glucose levels by inhibiting the activity of rate-limiting enzyme phosphofructokinase- 1 and slowing down glycolysis, while also activating fructose 1,6 bisphospatase to stimulate gluconeogenesis. Cytosolic citrate also downregulates fatty-acid $\beta$-oxidation pathways and is an allosteric activator of acetyl-CoA carboxylase beta isoform (ACCbeta), which affects transfer of fatty acids to mitochondria and its use in energy production. Reduced INDY transporting activity leads to low acetyl-CoA levels 
available for fatty acid and cholesterol synthesis, and also releases the inhibitory effects of citrate on glycolysis, thereby decreasing gluconeogenesis. Reduced INDY levels lead to reduced ATP production, and increased ADP levels that activate AMPK, a cell nutrient sensor. AMPK promotes insulin sensitivity, lipid-oxidation, and mitochondrial biogenesis by activating mitochondrial transcriptional co-activator $d P G C-1 /$ spargel $[18,47]$. AMPK also phosphorylates ACCbeta and inhibits its activation by citrate and its basal activity. Similar physiological changes are associated with adaptation to low nutrients during CR in a wide variety of species. Effects of Indy reduction on metabolism have been observed in flies, worms, mice, rats, non-human primates, and humans [10]. As noted in the previous section, reducing CeNAC-2, worm homologues of INDY lowers lipid levels, reduces weight, and activates AMPK [17]. $m I N D Y-K O$ mice have reduced hepatic lipogenesis, enhanced hepatic lipid oxidation, increased energy expenditure, increased mitochondrial biogenesis, increased insulin sensitivity, and are prevented from weight gain and protected from high-fat diet-induced steatosis and insulin resistance [18]. Adult rats aged on a high-fat diet have lower hepatic lipid levels and increased insulin sensitivity when liver-specific mINDY activity was reduced by using antisense oligonucleotides [39].

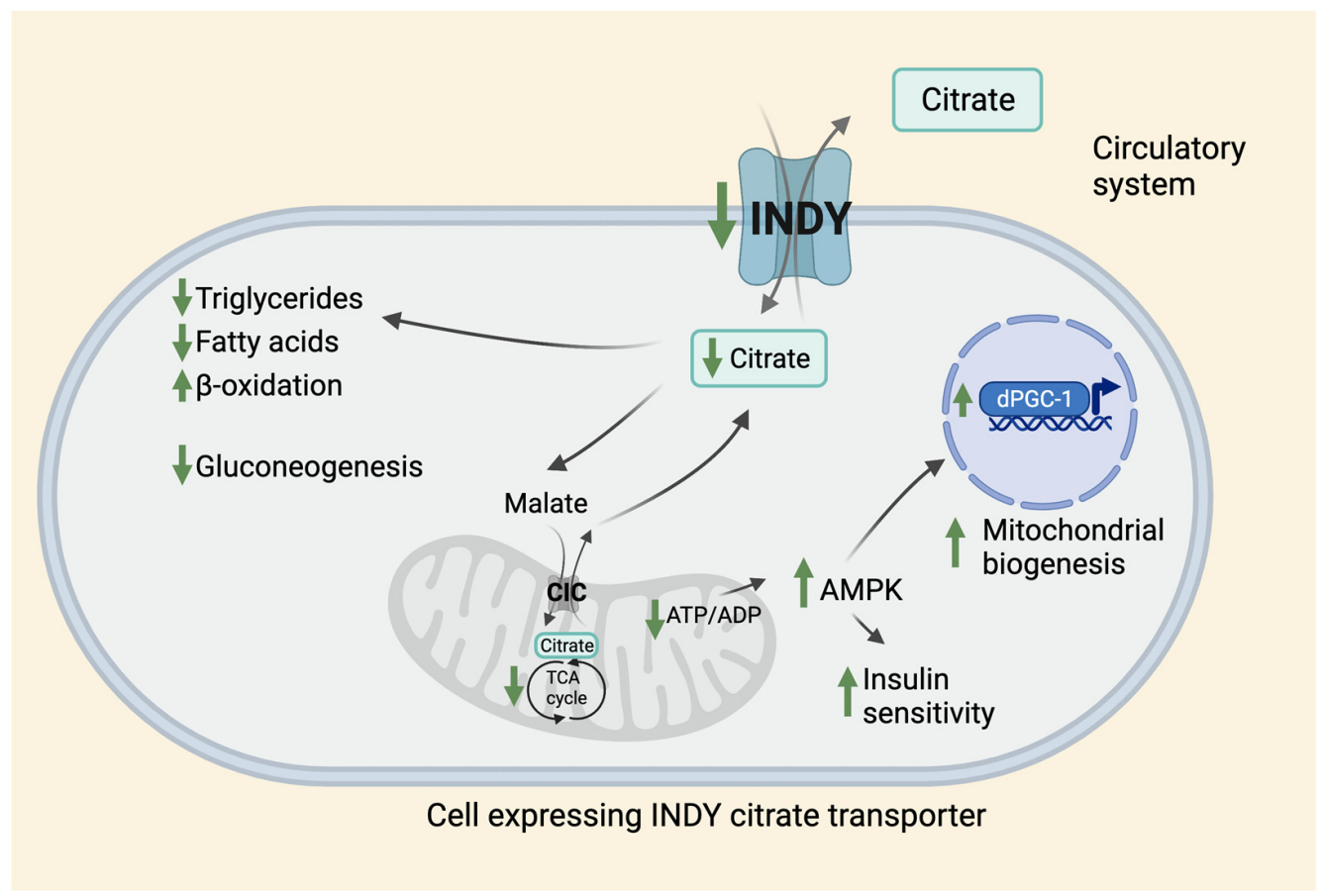

Figure 1. Effects of citrate transporter on metabolism. INDY is a plasma membrane citrate transporter that transports citrate between the circulatory system and the cytosol of the INDY-expressing cell in Drosophila and other species. Reduced INDY activity results in lower cellular levels of citrate that affects metabolism leading to reduced synthesis of triglycerides, and fatty acids, increased $\beta$-oxidation, and decreased gluconeogenesis. Citrate is converted to malate in the cytosol and is transported to mitochondria through CIC located on the inner mitochondrial membrane, where it is used in the TCA cycle. The citrate synthesized in the mitochondria is then transported back to the cytosol through CIC in exchange for malate. Low INDY levels lead to a decrease in ATP/ADP ratio that activates $\mathrm{AMPK}$, which promotes insulin sensitivity and mitochondrial biogenesis via upregulation of dPGC-1 levels. CIC: Citrate/isocitrate carrier.

The role of mINDY in metabolism is corroborated by severe phenotypes caused by mutations in mammalian mINDY. The mIndy gene encodes the only known neuronal plasma membrane citrate transporter expressed in the hippocampus, cerebellum, cerebral cortex, and olfactory bulb [20,48]. A loss of function of mIndy (mSLC13A5) and mutations in the coding region of the mIndy gene in humans is the cause of an autosomal recessive 
disorder referred to as infantile epileptic encephalopathy. This disorder causes neonatal epilepsy with seizures during the first few days of a newborn's life as well as developmental delays, limited speech, tooth defects, and limited motor skills $[49,50]$. There are about 41 different mutations in the mIndy gene, which are all associated with infantile epileptic encephalopathy $[9,11]$. Kohlschütter-Toönz syndrome (KTS) is another disorder caused by mIndy (mSLC13A5) mutations, which has similar phenotypes to infantile epileptic encephalopathy and the mechanism underlying KTS is unknown [51]. Mutations in mIndy are classified based on their effects on mINDY protein function as transporter or on its stability, but all mIndy mutations result in loss of citrate transport [50]. Lack of citrate transport affects neuronal energy production, neurotransmitter biosynthesis, as well as formation of the myelin sheath in neurons [11,19]. The original study on mINDY-KO mice did not find any abnormal phenotype that could suggest a seizure phenotype [18]. However, detailed examination of $m I N D Y$-KO mice using video-electroencephalography (EEG) monitoring showed that a fraction of $m I N D Y-K O$ mice had a propensity for epileptic seizures and proepileptic excitability changes [52]. In contrast, another recent report that used a different $m I N D Y-K O$ mouse model described increased motor coordination and improved recognition and social memory, which were similar to CR mice [53]. The role of mINDY in metabolism is further underscored by studies that show that silencing mINDY in human hepatocarcinoma cells decreases their proliferation [54-56].

\section{Indy Transcriptional Regulation}

The importance of citrate in metabolic regulation is underscored by reports that Indy transcription is regulated by nutritional status of the organism. Indy gene expression in the midgut is regulated by diet and stress. Indy mRNA levels are increased in aged control flies and in flies exposed to a high nutrient diet and oxidative stress. In contrast, control flies raised on a CR diet, and Indy $206 /+$ heterozygous and Ind $y^{206} / I n d y^{206}$ homozygous flies on a standard diet have reduced Indy mRNA levels [16,22]. In primary rat hepatocytes, mIndy transcription is induced during starvation by glucagon hormone via binding of the transcription factor cAMP-responsive element-binding protein (CREB) to its binding sequence in the Indy promoter [57]. This induction results in increased INDY transporting activity that leads to increased cytosolic citrate levels and its utilization in gluconeogenesis and lipid synthesis. Studies in human and rat primary liver cells have shown that mIndy transcription is upregulated by binding of rifampicin and the benzo[a]pyrene to the nuclear receptor pregnane $\mathrm{X}$ receptor (PXR) and aryl hydrocarbon receptor (Ahr), respectively $[58,59]$. Consistent with the metabolic role of INDY, increased mIndy expression levels lead to hepatic lipid accumulation. Furthermore, increased levels of mIndy expression are associated with non-alcoholic fatty liver disease (NAFLD) in obese, insulin-resistant humans [60]. This increase is caused by binding of the inflammatory interleukin-6 (IL-6), released from adipocytes, to the IL-6 receptor, which leads to phosphorylation and nuclear transfer of the transcriptional factor STAT3 and its binding to the STAT3 binding sequence in the mIndy promoter [60]. Moreover, inhibition of liver mIndy (mSLC13A5) by RNA interference (RNAi) prevents diet-induced NAFLD and improves insulin sensitivity in mice [40]. mIndy expression is upregulated in the liver cells of nonhuman primates fed for 2 years with a high-fat and high-sucrose diet. Their liver cells also had high levels of circulating lipids and glucose [60]. Taken together, INDY levels have profound effects on cellular metabolism that have been implicated in human NAFLD and obesity models in non-human primates and mice.

\section{Effects of Reduced Indy on Intestinal Stem Cell Homeostasis}

INDY is predominantly expressed in the basolateral membrane of the fly midgut, a tissue actively involved in metabolism. Therefore, it is essential to determine how INDY reduction affects intestinal stem cell (ISC) physiology and if these changes contribute to extended longevity. In flies and mammals, the gastrointestinal (GI) tract is involved in many physiological processes including nutrient digestion, nutrient absorption, protection 
against microbiota, endocrine function, and excretion [61]. The mammalian GI tract consists of ISCs that are self-renewing and give rise to progenitor cells that differentiate into several types of cells in the intestinal epithelium. Differentiating ISCs replace damaged cells to maintain gut integrity and function, which is vital for healthy aging $[62,63]$. The Drosophila gut has been an excellent system to study cellular mechanisms and biological processes involved in gut maintenance and homeostasis, due to similar physiological processes that occur in the intestines of flies and mammals [64]. The adult Drosophila gut has three regions: the foregut, midgut, and hindgut. The Drosophila midgut is analogous to the small intestine and contains multipotent ISCs, progenitor enteroblasts (EBs), enterocytes (ECs), and entero-endocrine cells (EEs) along with the visceral muscle (VM), which lines the intestinal epithelium along with the basement membrane. The EBs are post-mitotic cells that arise from the ISCs and differentiate into ECs, which have absorptive function and EEs, which have a role in secretion of gut hormones [65-67].

Aging is characterized by a gradual decrease in mitochondrial biogenesis caused by reduced expression of $d P G C-1 /$ spargel mRNA. dPGC-1 promotes mitochondrial biogenesis by activating expression of genes encoding mitochondrial proteins [16,68,69]. Ind $y^{206} /+$ and Ind $y^{206} /$ Indy ${ }^{206}$ flies have increased $d P G C-1$ mRNA levels in heads, thoraces, and midguts that lead to increased gene expression of electron transport chain (ETC) components and increased mitochondrial copy number (Figure 2) [16]. Indy $206 /+$ and Indy $206 /$ Indy 206 flies have lower levels of mitochondrial ROS production and increased expression levels of ROS-detoxification genes, Glutathione S transferase E1 (GstE1) and Glutathione S transferase D5 (GstD5), which contribute to their increased resistance to oxidative stress. Moreover, mitochondria isolated from the midguts of Indy $y^{206} /+$ and Indy $206 /$ Indy $y^{206}$ flies have high mitochondrial membrane potential that confirm preservation of mitochondrial function. Interventions that increase ETC function and regulate mitochondrial biogenesis through overexpression of $d P G C-1$ in the gut have led to preserved ISC homeostasis in older flies and extended lifespan in Drosophila [16,68-70]. Genetic epistasis studies showed that longevity was not extended in Indy ${ }^{206}$; $d P G C-1^{K G 08646}$ flies with both reduced Indy and $d P G C-1$ levels. In addition, overexpression of $d P G C-1$ in Indy $206 /+$ flies did not further extend the long life of Indy $206 /+$ flies, indicating that Indy and $d P G C-1$ longevity pathways overlap [16].

An age-associated increase in ROS levels in the midguts of flies activates ISC proliferation rate leading to uncontrolled hyperproliferation of ISCs and accumulation of mis-differentiated and damaged cells [61]. Indy $206 /+$ and Indy $206 /$ Indy $y^{206}$ flies have lower ROS levels compared to control flies, resulting in reduced ISC proliferation rate relative to controls at all ages, which was determined by the number of escargot-positive cells, a marker for ISCs and progenitor EBs (Figure 2). Actively proliferating cells in the midgut, measured as phosphorylated histone $\mathrm{H} 3$ ( $\mathrm{pH} 3$ )-positive cells, are also reduced in Indy flies indicating a delay in age-associated increase in ISC proliferation rate. These findings illustrate that Indy flies have preserved stem cell differentiation and ISC homeostasis [16]. It has been shown that CR prevents age-associated ISC hyperproliferation that contributes to extended lifespan [71]. It is possible that reduced Indy mRNA in CR flies contributes to preserved ISC homeostasis.

These studies suggest a link between ISC proliferation rate and Indy mRNA expression levels in response to nutrients, stress, and aging. It is highly likely that during aging and stress, ISCs have increased energy demands required for proliferation to replace aberrant cells, and a rise in INDY levels would increase cytoplasmic citrate levels that would be utilized for energy production in mitochondria. The role of mINDY in cell proliferation is supported by findings that RNAi mediated silencing of mINDY (mSLC13A5) in human hepatoma cell lines (HepG2) dramatically suppressed cell proliferation and colony formation $[54,56]$. The growth of HepG2 cell-derived xenographs containing mINDY-shRNA in nude mice was also decreased. Inhibitory effects of reduced mIndy contributed to decreased lipogenesis and decreased long-chain fatty acid and cholesterol synthesis, which are required for growth. Hepatocarcinoma proliferation suppression by mIndy inhibition 
may also be mediated by inhibitory effects of activated AMPK on the oncogenic mTOR signaling pathway [72].

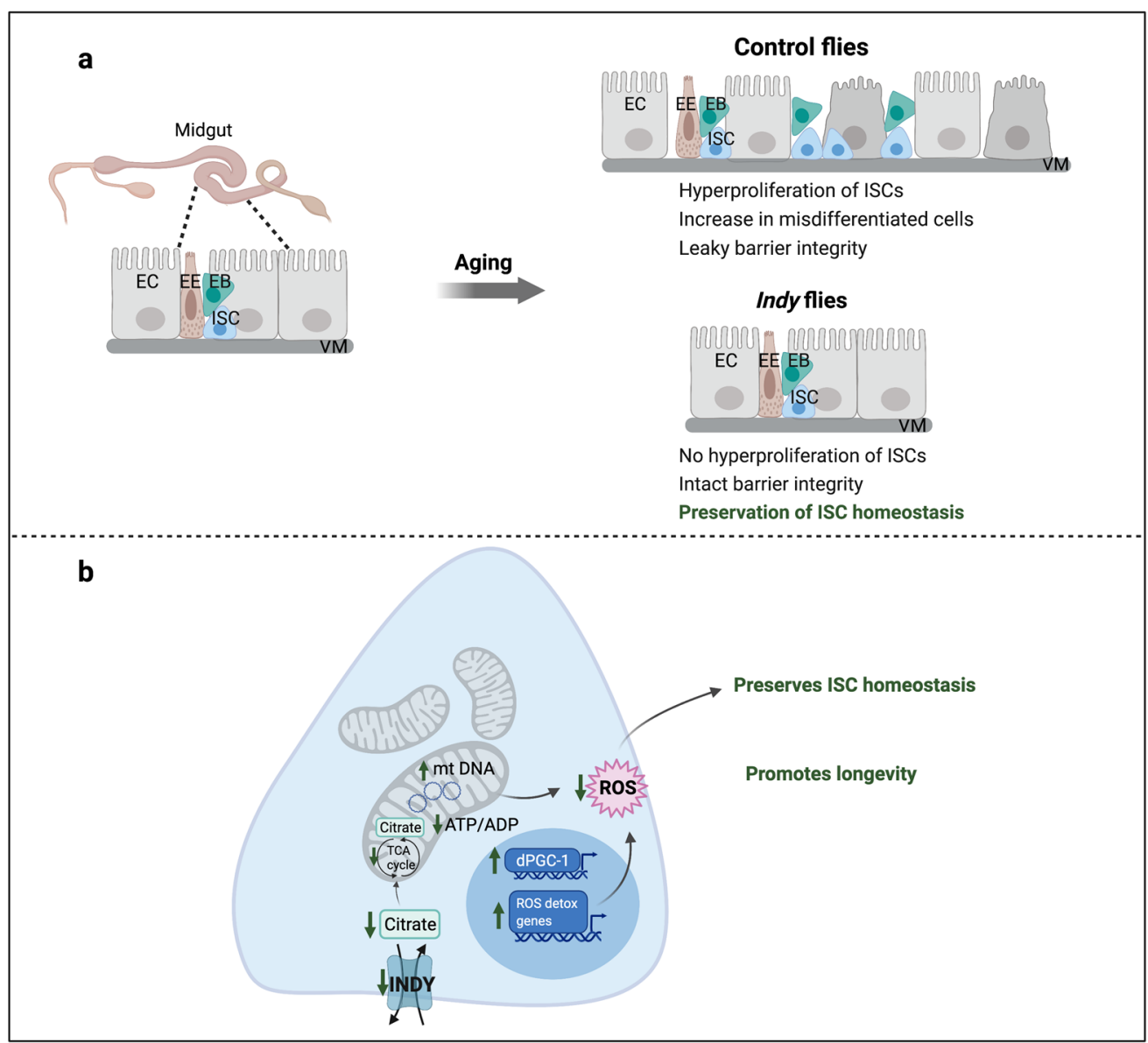

Figure 2. Indy reduction preserves ISC homeostasis. (a) Aging causes disruption of intestinal epithelium that results in loss of barrier integrity and uncontrolled proliferation of ISCs and accumulation of mis-differentiated ISCs resulting in loss of gut homeostasis; (b) Indy reduction in the midgut results in reduced cytosolic citrate levels, which increases mitochondrial biogenesis through upregulation of dPGC-1, increases mitochondrial DNA and number, and upregulates ROS-detoxification genes that reduces overall cellular ROS levels. This preserves ISC homeostasis and midgut integrity leading to a delay in intestinal aging thereby promoting longevity. ISC: Intestinal stem cell; EB: Enteroblast; EC: Enterocyte; EE: Enteroendocrine cell; VM: Visceral muscle; ROS: Reactive oxygen species.

With aging, there is a decline in the integrity of intestinal epithelium that acts as a barrier between intestinal lumen and hemolymph in the gut, and factors that maintain its structure and function are critical to promote longevity. Aged Indy ${ }^{206} /+$ and Indy $y^{206} /$ Indy $y^{206}$ flies exhibit intact intestinal structure and integrity revealed by electron micrographs. When these flies were fed with food containing non-absorptive blue dye, blue dye was only observed in the proboscis and gut [16]. In contrast, blue dye was observed in all tissues of control flies; because of a loss of intestinal integrity with aging, the dye can easily move from lumen of the gut to hemolymph and the rest of the body, resulting in what has been referred to as a "smurf" phenotype. Overall, this indicates that reducing INDY activity in the midgut preserves ISC homeostasis, enhances intestinal health, and promotes longevity. Molecular mechanisms that couple metabolism and ISC proliferation play a significant role in longevity, emphasizing the importance of future research to fully understand INDY's role as a link between these key processes. It is most likely that beneficial effects of reduced Indy on ISC homeostasis and metabolism in other tissues have a synergistic effect on fly health and longevity. While effects of Indy as well as mIndy reduction on metabolism in 
cells expressing INDY on their plasma membrane are highly conserved across species, mINDY is not present in the human GI tract. Therefore, it is highly likely that other SLC family transporters play the role of fly Indy in the human GI tract.

\section{Effects of Indy Reduction on Spermatogenesis}

A recent report by Hudry et al. highlights the role of INDY and citrate in interorgan communication and sperm maturation [73]. Interestingly, this communication is mediated by close spatial organization of fly testis and midgut. In Drosophila, the midgut consists of five distinct regions from anterior to posterior direction marked as R1-R5 [74,75]. JAK/STAT signaling is activated in the R4 midgut region by the cytokine Upd1 released from the testis soma and this has been shown to increase intestinal sugar gene expression to produce cytosolic citrate. INDY transports citrate from the R4 region to the testis and promotes maturation of sperm. Moreover, impaired citrate efflux in Indy knockdown flies negatively affects sperm maturation and decreases spermatocyte numbers by causing metabolic changes in testis. In males, citrate serves as a signal that regulates sex specific differences in sugar gene expression but also increases food uptake. Taken together, this study presents evidence that by affecting citrate levels INDY plays a role in interorgan communication between the gut and testis in a sex-dependent manner [73]. Therefore, it would be worth examining the role of mINDY in testis of mice, rats and humans, where INDY is expressed at low levels [10,19]. Effects of obesity, high-fat diet, and glucose and lipid metabolism, and the role of mitochondria on spermatogenesis in human males have been described [76,77].

The ability of Indy reduction to decrease spermatogenesis might seem contradictory to increased fecundity observed in Indy $206 /+$ and Indy $302 /+$ females $[1,23,73]$. However, we have previously examined a possibility that Indy reduction might affect male and female physiology differently, which could influence fecundity and longevity $[1,23]$. We determined fecundity of at least 23 mating pairs, each pair with a single male and female heterozygous for Indy $y^{206} /+$ and Indy $y^{302} /+$ and compared them to control flies (CS-5). Heterozygous F1 Ind $y^{206 /+}$ and Indy $302 /+$ flies were generated by two different crosses: the first where parental males were Ind $y^{206} / I n d y^{206}$ or Indy ${ }^{302} /$ Indy ${ }^{302}$ homozygous and females were CS-5 flies, and in the second where parental females were Indy homozygous flies (same lines) and males were CS-5 flies $[1,23]$. Heterozygous Ind $y^{206} /++$ and Indy $y^{302} /+$ flies, generated by both crossing schemes, laid more eggs compared to control flies with no significant difference between crosses [1]. No differences in metabolic rate or flight velocity were observed in INDY heterozygous flies from either crossing schemes [23]. Beneficial effects on female fecundity were also observed in three independently natural populations of flies containing the Hoppel element in the Indy gene region [24]. As noted in Section 2, while Indy $y^{206} /+$ and Indy $y^{302} /+$ fecundity was higher on a standard and high calorie diet, it was lower compared to controls when flies were kept on a CR diet, most likely due to low energy available for egg production, which is consistent with Indy reduction creating a state similar to CR [23].

It is worth noting that fly mating is a complex process that affects behavior, physiology, and longevity of female flies [78-80]. During mating, a male fly transfers hundreds of sperm to a female fly, who stores sperm in spermathecae and seminal receptacle for up to 2 weeks. Females are able to release sperm from storage and fertilize their own eggs for 2 weeks after a single mating. During mating, about 100 seminal fluid proteins (SFPs) are transferred together with sperm, which affect sperm storage, egg production, release of the sperm for fertilization, and other aspects of female physiology [78]. The numbers of spermatocytes were not determined in Indy $2^{206 /+}$ and Indy $y^{302 /+}$ flies; however, even if the number of sperm transferred from a male to female fly was lower compared to controls, the female flies would still likely have more than enough sperm to fertilize their eggs. Once the sperm and SFPs that prevent mating are depleted, female flies could mate again. In summary, while effects of Indy reduction on spermatogenesis and female egg production illustrate sex-specific effects, no other sex-specific differences were found in examined 
physiology, mortality rate or longevity but future detailed analyses in this area could reveal new insights.

\section{Conclusions and Future Directions}

Reduced gene expression of fly Indy and its worm homologues extends their life span by altering metabolism in a manner similar to CR. Fly INDY and homologues in worms and mammals share a preference for transporting citrate. By regulating cytoplasmic citrate levels, INDY acts as a metabolic regulator in modulating glucose and lipid levels, and energy production in mitochondria [44,81]. Metabolic changes associated with Indy reduction in the fly midgut results in dramatic changes in midgut physiology that lead to preserved intestinal ISC homeostasis. This is vital for replacement of damaged cells and the maintenance of midgut function illustrated by preserved intestinal integrity $[16,44]$. Indy reduction extends lifespan in male and female flies, but the effects of Indy reduction on ISC homeostasis have only been studied in female flies. Male and female flies have different gut pathologies and respond differently to stress and CR, with males having a delay in age-related gut pathology and lower ISC proliferation, while females respond better to stress and CR $[82,83]$. Considering these differences, it would be of interest to determine the effects of Indy reduction on the midguts of male flies. ISC homeostasis is regulated by multiple signaling pathways including IIS, Notch, EGF, Wnt/wingless, BMP/Dpp, JNK, and JAK/STAT, among others [62,84-86]. It would be important to assess the status of different signaling pathways in flies with reduced Indy expression, as metabolic changes might delay age-associated activation of these pathways and could contribute to preservation of ISC homeostasis and longevity. The data reviewed here support the role of INDY as a metabolic regulator: Indy expression changes in response to nutrient availability and requirements of the organism, which, by regulating citrate levels, controls energetic status of the organism to maintain tissue-specific metabolic requirements leading to preserved organismal health and homeostasis. Reduced INDY levels in the midgut could then prevent age-related ISC hyperproliferation by reducing the available energy for proliferation.

\section{INDY as a potential therapeutic target}

Aging can be modulated by genetic and environmental manipulations such as CR [87]. Reduced gene expression of fly Indy and its worm homologues extends their life span by altering metabolism in a manner similar to CR. Indy reduction decreases the rate of aging, as measured by a decrease in the slope of the mortality curve [23]. Thereby, pharmaceutical interventions that reduce INDY could mimic the benefits of CR without reducing caloric uptake. Studies in flies, worms, mice, and rats have made INDY a potential target that could be used in a clinical setting to alleviate some metabolic disorders such as NAFLD, Type 2 diabetes, insulin resistance, and even carcinogenesis. In brief, liver-specific mIndy RNAi silencing prevents diet-induced NAFLD in mice [40]; mINDY-KO mice are protected from age- and diet-induced insulin insensitivity and obesity; while antisense oligonucleotides given to rats on a high-fat diet prevent hepatic steatosis and hepatic insulin resistance [39]. Particularly promising is use of mINDY inhibitors in hepatocarcinoma cells due to their effects on energy required for proliferation and negative effects on oncogenic mTOR signaling. Recent studies in mINDY-KO mice indicate potential use of mINDY inhibitors for regulating blood pressure, increasing motor coordination, and promoting social and recognition memory in mice $[12,53,88]$. There is also an urgent need to study the mechanisms of negative effects of mIndy mutations in neonatal epilepsy in model organisms expressing $m I n d y$ human mutations to provide insights for future therapies. Altogether, the physiological function of INDY and its effects on metabolism are highly conserved across organisms, making INDY a compelling therapeutic target for preservation of metabolic homeostasis. 
Author Contributions: Conceptualization, K.K. and B.R.; investigation, K.K. and B.R.; resources, K.K. and B.R.; writing-original draft preparation, K.K. and B.R.; writing-review and editing, K.K. and B.R.; visualization, K.K. and B.R.; supervision, B.R.; funding acquisition, B.R. All authors have read and agreed to the published version of the manuscript.

Funding: This research was funded by the National Institute on Health, grant number RO1AG059586, R01AG059586-03S, UCONN REP, and by the University of Connecticut (UConn) Claude D. Pepper Older Americans Independence Center (P30-AG067988) to B.R., B.R. is a recipient of a Glenn Award for Research in Biological Mechanisms of Aging.

Acknowledgments: We thank Dushyant Mishra for helpful comments. We also thank Christopher Bonin and Geneva Hargis for stimulating discussion and providing beneficial edits. The figures were prepared using BioRender (https:/ / biorender.com/, accessed on 11 October 2021).

Conflicts of Interest: The authors declare no conflict of interest.

\section{References}

1. Rogina, B.; Reenan, R.A.; Nilsen, S.P.; Helfand, S.L. Extended Life-Span Conferred by Cotransporter Gene Mutations in Drosophila. Science 2000, 290, 2137-2140. [CrossRef]

2. Knauf, F.; Mohebbi, N.; Teichert, C.; Herold, D.; Rogina, B.; Helfand, S.; Gollasch, M.; Luft, F.C.; Aronson, P.S. The Life-Extending Gene Indy Encodes an Exchanger for Krebs-Cycle Intermediates. Biochem. J. 2006, 397, 25-29. [CrossRef]

3. Knauf, F.; Rogina, B.; Jiang, Z.; Aronson, P.S.; Helfand, S.L. Functional Characterization and Immunolocalization of the Transporter Encoded by the Life-Extending Gene Indy. Proc. Natl. Acad. Sci. USA 2002, 99, 14315-14319. [CrossRef]

4. Gopal, E.; Babu, E.; Ramachandran, S.; Bhutia, Y.D.; Prasad, P.D.; Ganapathy, V. Species-Specific Influence of Lithium on the Activity of SLC13A5 (NaCT): Lithium-Induced Activation Is Specific for the Transporter in Primates. J. Pharmacol. Exp. Ther. 2015, 353, 17-26. [CrossRef]

5. Wang, L.Y.; Jiang, J.; Ma, H.M. Molecular Characterization, Tissue Expression Profile, and SNP Analysis of Porcine SLC13A5. Genet. Mol. Res. 2015, 14, 16090-16101. [CrossRef]

6. Mancusso, R.; Gregorio, G.G.; Liu, Q.; Wang, D.-N. Structure and Mechanism of a Bacterial Sodium-Dependent Dicarboxylate Transporter. Nature 2012, 491, 622-626. [CrossRef] [PubMed]

7. Inoue, K.; Fei, Y.-J.; Zhuang, L.; Gopal, E.; Miyauchi, S.; Ganapathy, V. Functional Features and Genomic Organization of Mouse $\mathrm{NaCT}$, a Sodium-Coupled Transporter for Tricarboxylic Acid Cycle Intermediates. Biochem. J. 2004, 378, 949-957. [CrossRef] [PubMed]

8. Inoue, K.; Fei, Y.-J.; Huang, W.; Zhuang, L.; Chen, Z.; Ganapathy, V. Functional Identity of Drosophila melanogaster Indy as a Cation-Independent, Electroneutral Transporter for Tricarboxylic Acid-Cycle Intermediates. Biochem. J. 2002, 367, 313-319. [CrossRef] [PubMed]

9. Jaramillo-Martinez, V.; Urbatsch, I.L.; Ganapathy, V. Functional Distinction between Human and Mouse Sodium-Coupled Citrate Transporters and Its Biologic Significance: An Attempt for Structural Basis Using a Homology Modeling Approach. Chem. Rev. 2021, 121, 5359-5377. [CrossRef]

10. Willmes, D.M.; Kurzbach, A.; Henke, C.; Schumann, T.; Zahn, G.; Heifetz, A.; Jordan, J.; Helfand, S.L.; Birkenfeld, A.L. The Longevity Gene INDY (I'm Not Dead Yet) in Metabolic Control: Potential as Pharmacological Target. Pharmacol. Ther. 2018, 185, 1-11. [CrossRef]

11. Kopel, J.J.; Bhutia, Y.D.; Sivaprakasam, S.; Ganapathy, V. Consequences of NaCT/SLC13A5/mINDY Deficiency: Good versus Evil, Separated Only by the Blood-Brain Barrier. Biochem. J. 2021, 478, 463-486. [CrossRef] [PubMed]

12. Mishra, D.; Kannan, K.; Meadows, K.; Macro, J.; Li, M.; Frankel, S.; Rogina, B. INDY—From Flies to Worms, Mice, Rats, Non-Human Primates, and Humans. Front. Genet. 2021, 8, 66.

13. Rogina, B.; Helfand, S.L. INDY Mutations and Drosophila longevity. Front. Genet. 2013, 4, 47. [CrossRef] [PubMed]

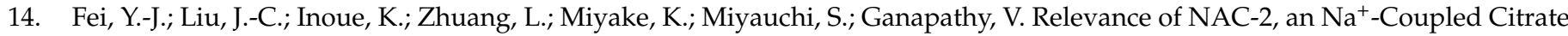
Transporter, to Life Span, Body Size and Fat Content in Caenorhabditis Elegans. Biochem. J. 2004, 379, 191-198. [CrossRef] [PubMed]

15. Fei, Y.-J.; Inoue, K.; Ganapathy, V. Structural and Functional Characteristics of Two Sodium-Coupled Dicarboxylate Transporters (CeNaDC1 and CeNaDC2) from Caenorhabditis Elegans and Their Relevance to Life Span. J. Biol. Chem. 2003, $278,6136-6144$. [CrossRef]

16. Rogers, R.P.; Rogina, B. Increased Mitochondrial Biogenesis Preserves Intestinal Stem Cell Homeostasis and Contributes to Longevity in Indy Mutant Flies. Aging (Albany NY) 2014, 6, 335-350. [CrossRef]

17. Schwarz, F.; Karadeniz, Z.; Fischer-Rosinsky, A.; Willmes, D.M.; Spranger, J.; Birkenfeld, A.L. Knockdown of Indy/CeNac2 Extends Caenorhabditis Elegans Life Span by Inducing AMPK/Aak-2. Aging 2015, 7, 553-567. [CrossRef]

18. Birkenfeld, A.L.; Lee, H.-Y.; Guebre-Egziabher, F.; Alves, T.C.; Jurczak, M.J.; Jornayvaz, F.R.; Zhang, D.; Hsiao, J.J.; MartinMontalvo, A.; Fischer-Rosinsky, A.; et al. Deletion of the Mammalian INDY Homolog Mimics Aspects of Dietary Restriction and Protects against Adiposity and Insulin Resistance in Mice. Cell Metab. 2011, 14, 184-195. [CrossRef] 
19. Bhutia, Y.D.; Kopel, J.J.; Lawrence, J.J.; Neugebauer, V.; Ganapathy, V. Plasma Membrane Na ${ }^{+}$-Coupled Citrate Transporter (SLC13A5) and Neonatal Epileptic Encephalopathy. Molecules 2017, 22, E378. [CrossRef]

20. Inoue, K.; Zhuang, L.; Maddox, D.M.; Smith, S.B.; Ganapathy, V. Structure, Function, and Expression Pattern of a Novel Sodium-Coupled Citrate Transporter (NaCT) Cloned from Mammalian Brain. J. Biol. Chem. 2002, 277, 39469-39476. [CrossRef]

21. Costello, L.C.; Franklin, R.B. Plasma Citrate Homeostasis: How It Is Regulated; And Its Physiological and Clinical Implications. An Important, But Neglected, Relationship in Medicine. HSOA J. Hum. Endocrinol. 2016, 1, 005. [CrossRef]

22. Wang, P.-Y.; Neretti, N.; Whitaker, R.; Hosier, S.; Chang, C.; Lu, D.; Rogina, B.; Helfand, S.L. Long-Lived Indy and Calorie Restriction Interact to Extend Life Span. Proc. Natl. Acad. Sci. USA. 2009, 106, 9262-9267. [CrossRef]

23. Marden, J.H.; Rogina, B.; Montooth, K.L.; Helfand, S.L. Conditional Tradeoffs between Aging and Organismal Performance of INDY Long-Lived Mutant Flies. Proc. Natl. Acad. Sci. USA. 2003, 100, 3369-3373. [CrossRef] [PubMed]

24. Zhu, C.-T.; Chang, C.; Reenan, R.A.; Helfand, S.L. Indy Gene Variation in Natural Populations Confers Fitness Advantage and Life Span Extension through Transposon Insertion. Aging (Albany NY) 2014, 6, 58-69. [CrossRef] [PubMed]

25. Rogina, B.; Benzer, S.; Helfand, S.L. Drosophila Drop-Dead Mutations Accelerate the Time Course of Age-Related Markers. Proc. Natl. Acad. Sci. USA 1997, 94, 6303-6306. [CrossRef] [PubMed]

26. Luckinbill, L.S.; Clare, M.J. Selection for Life Span in Drosophila melanogaster. Heredity 1985, 55, 9-18. [CrossRef]

27. Toivonen, J.M.; Walker, G.A.; Martinez-Diaz, P.; Bjedov, I.; Driege, Y.; Jacobs, H.T.; Gems, D.; Partridge, L. No Influence of Indy on Lifespan in Drosophila after Correction for Genetic and Cytoplasmic Background Effects. PLoS Genet. 2007, 3, e95. [CrossRef]

28. Evangelou, A.; Ignatiou, A.; Antoniou, C.; Kalanidou, S.; Chatzimatthaiou, S.; Shianiou, G.; Ellina, S.; Athanasiou, R.; Panagi, M.; Apidianakis, Y.; et al. Unpredictable Effects of the Genetic Background of Transgenic Lines in Physiological Quantitative Traits. G3 (Bethesda) 2019, 9, 3877-3890. [CrossRef]

29. Tamamouna, V.; Panagi, M.; Theophanous, A.; Demosthenous, M.; Michail, M.; Papadopoulou, M.; Teloni, S.; Pitsouli, C.; Apidianakis, Y. Evidence of Two Types of Balance between Stem Cell Mitosis and Enterocyte Nucleus Growth in the Drosophila Midgut. Development 2020, 147, dev189472. [CrossRef] [PubMed]

30. Bross, T.G.; Rogina, B.; Helfand, S.L. Behavioral, Physical, and Demographic Changes in Drosophila Populations through Dietary Restriction. Aging Cell 2005, 4, 309-317. [CrossRef]

31. Parashar, V.; Rogina, B. dSir2 Mediates the Increased Spontaneous Physical Activity in Flies on Calorie Restriction. Aging 2009, 1, 529-541. [CrossRef]

32. Neretti, N.; Wang, P.-Y.; Brodsky, A.S.; Nyguyen, H.H.; White, K.P.; Rogina, B.; Helfand, S.L. Long-Lived Indy Induces Reduced Mitochondrial Reactive Oxygen Species Production and Oxidative Damage. Proc. Natl. Acad. Sci. USA 2009, 106, $2277-2282$. [CrossRef]

33. Kenyon, C.; Chang, J.; Gensch, E.; Rudner, A.; Tabtiang, R. A C. Elegans Mutant That Lives Twice as Long as Wild Type. Nature 1993, 366, 461-464. [CrossRef]

34. Clancy, D.J.; Gems, D.; Harshman, L.G.; Oldham, S.; Stocker, H.; Hafen, E.; Leevers, S.J.; Partridge, L. Extension of Life-Span by Loss of CHICO, a Drosophila Insulin Receptor Substrate Protein. Science 2001, 292, 104-106. [CrossRef] [PubMed]

35. Tatar, M.; Kopelman, A.; Epstein, D.; Tu, M.P.; Yin, C.M.; Garofalo, R.S. A Mutant Drosophila Insulin Receptor Homolog That Extends Life-Span and Impairs Neuroendocrine Function. Science 2001, 292, 107-110. [CrossRef] [PubMed]

36. Kannan, K.; Fridell, Y.-W.C. Functional Implications of Drosophila Insulin-like Peptides in Metabolism, Aging, and Dietary Restriction. Front. Physiol. 2013, 4, 288. [CrossRef] [PubMed]

37. Nässel, D.R.; Liu, Y.; Luo, J. Insulin/IGF Signaling and Its Regulation in Drosophila. Gen. Comp. Endocrinol. 2015, 221, 255-266. [CrossRef] [PubMed]

38. Gross, D.N.; van den Heuvel, A.P.J.; Birnbaum, M.J. The Role of FoxO in the Regulation of Metabolism. Oncogene 2008, 27, 2320-2336. [CrossRef] [PubMed]

39. Pesta, D.H.; Perry, R.J.; Guebre-Egziabher, F.; Zhang, D.; Jurczak, M.; Fischer-Rosinsky, A.; Daniels, M.A.; Willmes, D.M.; Bhanot, S.; Bornstein, S.R.; et al. Prevention of Diet-Induced Hepatic Steatosis and Hepatic Insulin Resistance by Second Generation Antisense Oligonucleotides Targeted to the Longevity Gene mINDY (Slc13a5). Aging (Albany NY) 2015, 7, 1086-1093. [CrossRef]

40. Brachs, S.; Winkel, A.F.; Tang, H.; Birkenfeld, A.L.; Brunner, B.; Jahn-Hofmann, K.; Margerie, D.; Ruetten, H.; Schmoll, D.; Spranger, J. Inhibition of Citrate Cotransporter Slc13a5/mINDY by RNAi Improves Hepatic Insulin Sensitivity and Prevents Diet-Induced Non-Alcoholic Fatty Liver Disease in Mice. Mol. Metab. 2016, 5, 1072-1082. [CrossRef]

41. Gopal, E.; Miyauchi, S.; Martin, P.M.; Ananth, S.; Srinivas, S.R.; Smith, S.B.; Prasad, P.D.; Ganapathy, V. Expression and Functional Features of NaCT, a Sodium-Coupled Citrate Transporter, in Human and Rat Livers and Cell Lines. Am. J. Physiol. Gastrointest Liver Physiol. 2007, 292, G402-G408. [CrossRef]

42. Ruderman, N.B.; Saha, A.K.; Vavvas, D.; Witters, L.A. Malonyl-CoA, Fuel Sensing, and Insulin Resistance. Am. J. Physiol. 1999, 276, E1-E18. [CrossRef]

43. Rogers, R.P.; Rogina, B. The Role of INDY in Metabolism, Health and Longevity. Front. Genet. 2015, 6. [CrossRef]

44. Rogina, B. INDY-A New Link to Metabolic Regulation in Animals and Humans. Front. Genet. 2017, 8, 66. [CrossRef] [PubMed]

45. Ferramosca, A.; Zara, V. Dietary Fat and Hepatic Lipogenesis: Mitochondrial Citrate Carrier as a Sensor of Metabolic Changes. Adv. Nutr. 2014, 5, 217-225. [CrossRef] [PubMed]

46. Sun, J.; Aluvila, S.; Kotaria, R.; Mayor, J.A.; Walters, D.E.; Kaplan, R.S. Mitochondrial and Plasma Membrane Citrate Transporters: Discovery of Selective Inhibitors and Application to Structure/Function Analysis. Mol. Cell Pharmacol. 2010, 2, 101-110. 
47. Zhang, J.; Nuebel, E.; Daley, G.Q.; Koehler, C.M.; Teitell, M.A. Metabolic Regulation in Pluripotent Stem Cells during Reprogramming and Self-Renewal. Cell Stem Cell 2012, 11, 589-595. [CrossRef] [PubMed]

48. Pajor, A.M.; Gangula, R.; Yao, X. Cloning and Functional Characterization of a High-Affinity Na $\left(^{+}\right) /$Dicarboxylate Cotransporter from Mouse Brain. Am. J. Physiol. Cell Physiol. 2001, 280, C1215-C1223. [CrossRef]

49. Thevenon, J.; Milh, M.; Feillet, F.; St-Onge, J.; Duffourd, Y.; Jugé, C.; Roubertie, A.; Héron, D.; Mignot, C.; Raffo, E.; et al. Mutations in SLC13A5 Cause Autosomal-Recessive Epileptic Encephalopathy with Seizure Onset in the First Days of Life. Am. J. Hum. Genet. 2014, 95, 113-120. [CrossRef] [PubMed]

50. Hardies, K.; de Kovel, C.G.F.; Weckhuysen, S.; Asselbergh, B.; Geuens, T.; Deconinck, T.; Azmi, A.; May, P.; Brilstra, E.; Becker, F.; et al. Recessive Mutations in SLC13A5 Result in a Loss of Citrate Transport and Cause Neonatal Epilepsy, Developmental Delay and Teeth Hypoplasia. Brain 2015, 138, 3238-3250. [CrossRef]

51. Schossig, A.; Bloch-Zupan, A.; Lussi, A.; Wolf, N.I.; Raskin, S.; Cohen, M.; Giuliano, F.; Jurgens, J.; Krabichler, B.; Koolen, D.A.; et al. SLC13A5 Is the Second Gene Associated with Kohlschütter-Tönz Syndrome. J. Med. Genet. 2017, 54, 54-62. [CrossRef]

52. Henke, C.; Töllner, K.; van Dijk, R.M.; Miljanovic, N.; Cordes, T.; Twele, F.; Bröer, S.; Ziesak, V.; Rohde, M.; Hauck, S.M.; et al. Disruption of the Sodium-Dependent Citrate Transporter SLC13A5 in Mice Causes Alterations in Brain Citrate Levels and Neuronal Network Excitability in the Hippocampus. Neurobiol. Dis. 2020, 143, 105018. [CrossRef]

53. Fan, S.-Z.; Sung, C.-W.; Tsai, Y.-H.; Yeh, S.-R.; Lin, W.-S.; Wang, P.-Y. Nervous System Deletion of Mammalian INDY in Mice Mimics Dietary Restriction-Induced Memory Enhancement. J. Gerontol. Ser. A 2021, 76, 50-56. [CrossRef]

54. Li, Z.; Li, D.; Choi, E.Y.; Lapidus, R.; Zhang, L.; Huang, S.-M.; Shapiro, P.; Wang, H. Silencing of Solute Carrier Family 13 Member 5 Disrupts Energy Homeostasis and Inhibits Proliferation of Human Hepatocarcinoma Cells. J. Biol. Chem. 2017, 292, 13890-13901. [CrossRef] [PubMed]

55. Kumar, A.; Cordes, T.; Thalacker-Mercer, A.E.; Pajor, A.M.; Murphy, A.N.; Metallo, C.M. NaCT/SLC13A5 Facilitates Citrate Import and Metabolism under Nutrient-Limited Conditions. Cell Rep. 2021, 36, 109701. [CrossRef] [PubMed]

56. Eniafe, J.; Jiang, S. The Functional Roles of TCA Cycle Metabolites in Cancer. Oncogene 2021, 40, 3351-3363. [CrossRef] [PubMed]

57. Neuschäfer-Rube, F.; Lieske, S.; Kuna, M.; Henkel, J.; Perry, R.J.; Erion, D.M.; Pesta, D.; Willmes, D.M.; Brachs, S.; von Loeffelholz, C.; et al. The Mammalian INDY Homolog Is Induced by CREB in a Rat Model of Type 2 Diabetes. Diabetes 2014, 63, $1048-1057$. [CrossRef] [PubMed]

58. Li, L.; Li, H.; Garzel, B.; Yang, H.; Sueyoshi, T.; Li, Q.; Shu, Y.; Zhang, J.; Hu, B.; Heyward, S.; et al. SLC13A5 Is a Novel Transcriptional Target of the Pregnane X Receptor and Sensitizes Drug-Induced Steatosis in Human Liver. Mol. Pharmacol. 2015, 87, 674-682. [CrossRef]

59. Neuschäfer-Rube, F.; Schraplau, A.; Schewe, B.; Lieske, S.; Krützfeldt, J.-M.; Ringel, S.; Henkel, J.; Birkenfeld, A.L.; Püschel, G.P. Arylhydrocarbon Receptor-Dependent mINDY (Slc13a5) Induction as Possible Contributor to Benzo[a]Pyrene-Induced Lipid Accumulation in Hepatocytes. Toxicology 2015, 337, 1-9. [CrossRef]

60. von Loeffelholz, C.; Lieske, S.; Neuschäfer-Rube, F.; Willmes, D.M.; Raschzok, N.; Sauer, I.M.; König, J.; Fromm, M.F.; Horn, P.; Chatzigeorgiou, A.; et al. The Human Longevity Gene Homolog INDY and Interleukin-6 Interact in Hepatic Lipid Metabolism: Liver Biology/Pathobiology. Hepatology 2017, 66, 616-630. [CrossRef] [PubMed]

61. Rodriguez-Fernandez, I.A.; Tauc, H.M.; Jasper, H. Hallmarks of Aging Drosophila Intestinal Stem Cells. Mech. Ageing Dev. 2020, 190, 111285. [CrossRef]

62. Jasper, H. Intestinal Stem Cell Aging: Origins and Interventions. Annu. Rev. Physiol. 2020, 82, 203-226. [CrossRef]

63. Funk, M.C.; Zhou, J.; Boutros, M. Ageing, Metabolism and the Intestine. EMBO Rep. 2020, 21. [CrossRef]

64. Edgecomb, R.S.; Harth, C.E.; Schneiderman, A.M. Regulation of Feeding Behavior in Adult Drosophila melanogaster Varies with Feeding Regime and Nutritional State. J. Exp. Biol 1994, 197, 215-235. [CrossRef]

65. Micchelli, C.A.; Perrimon, N. Evidence That Stem Cells Reside in the Adult Drosophila Midgut Epithelium. Nature 2006, 439, 475-479. [CrossRef] [PubMed]

66. Ohlstein, B.; Spradling, A. The Adult Drosophila Posterior Midgut Is Maintained by Pluripotent Stem Cells. Nature 2006, 439, 470-474. [CrossRef] [PubMed]

67. Jiang, H.; Edgar, B.A. Intestinal Stem Cell Function in Drosophila and Mice. Curr. Opin. Genet. Dev. 2012, 22, 354-360. [CrossRef]

68. Hur, J.H.; Bahadorani, S.; Graniel, J.; Koehler, C.L.; Ulgherait, M.; Rera, M.; Jones, D.L.; Walker, D.W. Increased Longevity Mediated by Yeast NDI1 Expression in Drosophila Intestinal Stem and Progenitor Cells. Aging (Albany NY) $2013,5,662-681$. [CrossRef] [PubMed]

69. Rera, M.; Bahadorani, S.; Cho, J.; Koehler, C.L.; Ulgherait, M.; Hur, J.H.; Ansari, W.S.; Lo, T.; Jones, D.L.; Walker, D.W. Modulation of Longevity and Tissue Homeostasis by the Drosophila PGC-1 Homolog. Cell Metab. 2011, 14, 623-634. [CrossRef]

70. Rogers, R.P.; Rogina, B. A Gutsy Way to Extend Longevity. Front. Gene. 2012, 3, 108. [CrossRef] [PubMed]

71. Akagi, K.; Wilson, K.A.; Katewa, S.D.; Ortega, M.; Simons, J.; Hilsabeck, T.A.; Kapuria, S.; Sharma, A.; Jasper, H.; Kapahi, P. Dietary Restriction Improves Intestinal Cellular Fitness to Enhance Gut Barrier Function and Lifespan in D. Melanogaster. PLoS Genet. 2018, 14, e1007777. [CrossRef]

72. Peters, J.M. Flipping a Citrate Switch on Liver Cancer Cells. J. Biol. Chem. 2017, 292, 13902-13903. [CrossRef] [PubMed]

73. Hudry, B.; de Goeij, E.; Mineo, A.; Gaspar, P.; Hadjieconomou, D.; Studd, C.; Mokochinski, J.B.; Kramer, H.B.; Plaçais, P.-Y.; Preat, T.; et al. Sex Differences in Intestinal Carbohydrate Metabolism Promote Food Intake and Sperm Maturation. Cell 2019, 178, 901-918.e16. [CrossRef] [PubMed] 
74. Buchon, N.; Osman, D.; David, F.P.A.; Fang, H.Y.; Boquete, J.-P.; Deplancke, B.; Lemaitre, B. Morphological and Molecular Characterization of Adult Midgut Compartmentalization in Drosophila. Cell Rep. 2013, 3, 1725-1738. [CrossRef]

75. Marianes, A.; Spradling, A.C. Physiological and Stem Cell Compartmentalization within the Drosophila Midgut. eLife 2013, 2, e00886. [CrossRef] [PubMed]

76. Oliveira, P.F.; Sousa, M.; Silva, B.M.; Monteiro, M.P.; Alves, M.G. Obesity, Energy Balance and Spermatogenesis. Reproduction 2017, 153, R173-R185. [CrossRef]

77. Luo, D.; Zhang, M.; Su, X.; Liu, L.; Zhou, X.; Zhang, X.; Zheng, D.; Yu, C.; Guan, Q. High Fat Diet Impairs Spermatogenesis by Regulating Glucose and Lipid Metabolism in Sertoli Cells. Life Sci. 2020, 257, 118028. [CrossRef]

78. Neubaum, D.M.; Wolfner, M.F. 3 Wise, Winsome, or Weird? Mechanisms of Sperm Storage in Female Animals. Curr. Top. Dev. Biol. 1998, 41, 67-97.

79. Rogina, B.; Wolverton, T.; Bross, T.G.; Chen, K.; Müller, H.-G.; Carey, J.R. Distinct Biological Epochs in the Reproductive Life of Female Drosophila melanogaster. Mech. Ageing Dev. 2007, 128, 477-485. [CrossRef]

80. Rogina, B. The Effect of Sex Peptide and Calorie Intake on Fecundity in Female Drosophila melanogaster. Sci. World J. 2009, 9, 1178-1189. [CrossRef]

81. Frankel, S.; Rogina, B. INDY Mutants: Live Long and Prosper. Front. Genet. 2012, 3, 13. [CrossRef] [PubMed]

82. Regan, J.C.; Khericha, M.; Dobson, A.J.; Bolukbasi, E.; Rattanavirotkul, N.; Partridge, L. Sex Difference in Pathology of the Ageing Gut Mediates the Greater Response of Female Lifespan to Dietary Restriction. Elife 2016, 5, e10956. [CrossRef] [PubMed]

83. Millington, J.W.; Rideout, E.J. Sex Differences in Drosophila Development and Physiology. Curr. Opin. Physiol. 2018, 6, 46-56. [CrossRef]

84. Choi, N.-H.; Kim, J.-G.; Yang, D.-J.; Kim, Y.-S.; Yoo, M.-A. Age-Related Changes in Drosophila Midgut Are Associated with PVF2, a PDGF/VEGF-like Growth Factor. Aging Cell 2008, 7, 318-334. [CrossRef] [PubMed]

85. Biteau, B.; Karpac, J.; Hwangbo, D.; Jasper, H. Regulation of Drosophila Lifespan by JNK Signaling. Exp. Gerontology 2011, 46, 349-354. [CrossRef]

86. Jiang, H.; Patel, P.H.; Kohlmaier, A.; Grenley, M.O.; McEwen, D.G.; Edgar, B.A. Cytokine/Jak/Stat Signaling Mediates Regeneration and Homeostasis in the Drosophila Midgut. Cell 2009, 137, 1343-1355. [CrossRef]

87. Helfand, S.L.; Rogina, B. From Genes to Aging in Drosophila. In Advances in Genetics; Elsevier: Amsterdam, The Netherlands, 2003; Volume 49, pp. 67-109, ISBN 978-0-12-017649-6.

88. Willmes, D.M.; Daniels, M.; Kurzbach, A.; Lieske, S.; Bechmann, N.; Schumann, T.; Henke, C.; El-Agroudy, N.N.; Da Costa Goncalves, A.C.; Peitzsch, M.; et al. The Longevity Gene mINDY (I'm Not Dead, Yet) Affects Blood Pressure through Sympathoadrenal Mechanisms. JCI Insight 2021, 6, e136083. [CrossRef] 